TRANSACTIONS OF THE

AMERICAN MATHEMATICAL SOCIETY

Volume 172, October 1972

\title{
MULTIPLIERS ON MODULES OVER THE FOURIER ALGEBRA(1)
}

BY

\section{CHARLES F. DUNKL AND DONALD E. RAMIREZ}

ABSTRACT. Let $G$ be an infinite compact group and $\hat{G}$ its dual. For $1 \leq p<\infty, \mathfrak{L}^{p}(\hat{G})$ is a module over $\mathfrak{L}^{1}(\hat{G}) \cong A(G)$, the Fourier algebra of $G$. For $1 \leq p, q<\infty$, let $\pi_{p, q}=\operatorname{Hom}_{A(G)}\left(\mathfrak{\complement}^{p}(\hat{G}), \mathfrak{L}^{q}(\hat{G})\right)$. If $G$ is abelian, then $\pi_{p, p}$ is the space of $L^{P}(\hat{G})$-multipliers. For $1 \leq p<2$ and $p^{\prime}$ the conjugate index of $p$,

$$
A(G) \cong \pi_{1,1} \subset \pi_{p, p}=\pi_{p^{\prime}, p^{\prime}} \varsubsetneqq \pi_{2,2} \cong L^{\infty}(G) .
$$

Further, the space $\pi_{p, p}$ is the dual of a space called $Q_{p}$, a subspace of $\mathfrak{C}_{0}(\hat{G})$. Using a method of J. F. Price we observe that

$$
\bigcup\left\{\pi_{q, q}: 1 \leq q<p\right\} \subsetneq \pi_{p, p} \subsetneq \bigcap\left\{\pi_{q, q}: p<q<2\right\}
$$

(where $1<p<2$ ). Finally, $\pi_{q, p}=\{0\}$ for $1 \leq p<q<\infty$.

1. Modules, over the Fourier algebra. Throughout this paper $G$ will denote an infinite compact group and $\hat{G}$ its dual (we use the notation from [1]). Throughout, $1 \leq p, q, r \leq \infty$. Given $p$, the conjugate index will be denoted by $p^{\prime}\left(1 / p+1 / p^{\prime}=1\right)$.

Definition. Let $\phi \in \mathcal{C}_{F}(\hat{G})$ and so $\phi=\hat{f}$ for $f$ a trigonometric polynomial on $G$. We define $\check{\phi}$ by the rule $\check{\phi}=(\check{f})^{\wedge}$ where $\breve{f}(x)=f\left(x^{-1}\right), x \in G$.

Proposition 1. The map $\phi \mapsto \check{\phi}$ from $\mathcal{C}_{F}(\hat{G})$ to $\mathcal{C}_{F}(\hat{G})$ extends to an isometry of $\mathcal{L}^{p}(\hat{G}) \quad(1 \leq p<\infty)$ and of $\mathcal{C}_{0}(\hat{G})$.

Proof. For $f$ a trigonometric polynomial on $G$, we have that $(\check{f})^{\wedge}=\left((\bar{f})^{*}\right)^{\wedge}=$ $\left((\bar{f})^{\wedge}\right)^{*}=(J \hat{f} J)^{*}$ (see $\left[1\right.$, p. 87]). Thus for $\phi \in \mathcal{C}_{F}(\hat{G}),\|\check{\phi}\|_{p}=\|\phi\|_{p}$.

Definition. Let $\phi, \psi \in \mathcal{C}_{F}(\hat{G})$, we define $\phi \times \psi \in \mathcal{C}_{F}(\hat{G})$ by the rule $(\phi \times \psi)^{\wedge}=\hat{\phi} \hat{\psi}(\hat{\phi}$ denotes the inverse Fourier transform of $\phi[1$, p. 97]). We note that $\|\phi \times \psi\|_{1} \leq\|\phi\|_{1}\|\psi\|_{1}, \phi, \psi \in \mathcal{C}_{F}(\hat{G})$ (see $\left.[1, \mathrm{p} .93]\right)$. We define the pairing $\langle\phi, \psi\rangle=\operatorname{Tr}(\phi \breve{\psi})=\left(\ddot{\phi} *(\breve{\psi})^{\wedge}\right)(e)=\int_{G} \hat{\phi}(x) \hat{\psi}(x) d m_{G}(x), \phi, \psi \in \mathcal{C}_{F}(\hat{G})$,

Presented to the Society, October 18, 1971; received by the editors November 3,1971. 46L20.

AMS (MOS) subject classifications (1970). Primary 43A15, 43A22; Secondary 46E30,

Key words and phrases. Fourier algebra, modules over the Fourier algebra, multipliers.

(1) This research was supported in part by NSF contract numbers GP-19852 and GP-31483X.

Copyright $\odot 1973$, American Mathematical Society 
$e$ the identity in $G$. Equivalently, $\langle\phi, \psi\rangle=(\phi \times \psi)_{\iota}$ (where $\iota$ denotes the trivial representation $x \mapsto 1: G \rightarrow C)$.

The map $(\phi, \psi) \mapsto\langle\phi, \psi\rangle$ extends to a pairing between $\mathcal{L}^{\phi}(\hat{G})$ and $\mathcal{Q}^{\prime}(\hat{G})$ $(1 \leq p<\infty)$, that is, $|\langle\phi, \psi\rangle| \leq\|\phi\|_{p}\|\psi\|_{p^{\prime}}$, and $\|\phi\|_{p}=\sup \left\{|\langle\phi, \psi\rangle|:\|\psi\|_{p^{\prime}} \leq 1\right\}$, $\phi, \psi \in \mathcal{C}_{F}(\hat{G})$ (see $[1$, p. 144]).

Theorem 2. For $1 / p+1 / q \geq 1$, the map $(\phi, \psi) \mapsto \phi \times \psi: \mathcal{C}_{F}(\hat{G}) \times \mathcal{C}_{F}(\hat{G})$ $\rightarrow \mathcal{C}_{F}(\hat{G})$ extends to a map of $\mathfrak{L}^{p}(\hat{G}) \times \mathfrak{Q}^{q}(\hat{G}) \rightarrow \mathcal{P r}^{r}(\hat{G}), 1 / r=1 / p+1 / q-1$ (we replace $\mathfrak{\complement}^{\infty}(\hat{G})$ by $\left.\mathcal{C}_{0}(\hat{G})\right)$, such that $\|\phi \times \psi\|_{r} \leq\|\phi\|_{p}\|\psi\|_{q}, \phi \in \mathfrak{\complement}^{p}(\hat{G})$, $\psi \in \mathscr{L}^{q}(\hat{G})$.

Proof. For $\phi, \psi, \theta \in \mathcal{C}_{F}(\hat{G})$ we define the form $F$ on $\mathcal{C}_{F}(\hat{G}) \times \mathcal{C}_{F}(\hat{G}) \times$ $\mathcal{C}_{F}(\hat{G})$ by the rule $F(\phi, \psi, \theta)=\langle\phi \times \psi, \theta\rangle=\int_{G} \hat{\phi}(x) \hat{\psi}(x) \hat{\theta}(x) d m_{G}(x)=\langle\psi, \phi \times \theta\rangle$; and thus $F$ is symmetric. Now $|F(\phi, \psi, \theta)| \leq\|\phi \times \psi\|_{1}\|\theta\|_{\infty} \leq\|\phi\|_{1}\|\psi\|_{1}\|\theta\|_{\infty}$, $\phi, \psi, \theta \in \mathcal{C}_{F}(\hat{G})$. Let

$$
M\left(a_{1}, a_{2}, a_{3}\right)=\sup \left\{\left|F\left(\phi_{1}, \phi_{2}, \phi_{3}\right)\right|: \phi_{j} \in \mathcal{C}_{F}(\hat{G}),\left\|\phi_{j}\right\|_{1 / a_{j}} \leq 1,1 \leq j \leq 3\right\},
$$

$a_{1}, a_{2}, a_{3} \in[0,1]$. By the Riesz-Thorin convexity theorem for integration algebras $[1$, p. 143], it follows that $\log M$ is a convex function on $[0,1] \times$ $[0,1] \times[0,1]$. Since $M(1,0,1), M(1,1,0), M(0,1,1) \leq 1$, it follows by interpolating that $M\left(1 / p, 1 / q, 1 / r^{\prime}\right) \leq 1$ where $1 / r=1 / p+1 / q-1$.

Corollary 3. For $1 \leq p<\infty, \mathfrak{L}^{1}(\hat{G}) \times \mathfrak{L}^{p}(\hat{G})=\mathfrak{L}^{p}(\hat{G})$ and so $\mathfrak{L}^{p}(\hat{G})$ is an $\mathscr{L}^{1}(\hat{G})$-module. Also $\mathfrak{L}^{1}(\hat{G}) \times \mathcal{C}_{0}(\hat{G})=\mathcal{C}_{0}(\hat{G})$. For $1<p<\infty, \mathfrak{L}^{p}(\hat{G}) \times \mathfrak{L}^{p^{\prime}}(\hat{G}) \subset$ $\mathcal{C}_{0}(\hat{G})$. For $1 / p+1 / q>1, \mathfrak{L}^{p}(\hat{G}) \times \mathfrak{Q}^{q}(\hat{G}) \subset \mathfrak{Q}^{\operatorname{ar}}(\hat{G}), 1 / r=1 / p+1 / q-1$.

Theorem 4. $\mathfrak{L}^{2}(\hat{G}) \times \mathfrak{\complement}^{2}(\hat{G})=L^{1}(G)^{\wedge}$.

Proof. Let $\phi, \psi \in \mathfrak{L}^{2}(\hat{G})$ and choose $\left\{f_{n}\right\}_{n=1}^{\infty},\left\{g_{n}\right\}_{n=1}^{\infty}$ sequences of trigonometric polynomials on $G$ such that $\hat{f}_{n} \stackrel{n}{\rightarrow} \phi, \hat{g}_{n} \stackrel{n}{\rightarrow} \psi$ in $\mathfrak{L}^{2}(\hat{G})$. Then $f_{n} g_{n} \in L^{1}(G)$, and we wish to show that $\phi \times \psi=\lim _{n \rightarrow \infty} \hat{f}_{n} \times \hat{g}_{n}=\lim _{n \rightarrow \infty}\left(f_{n} g_{n}\right)^{\wedge}$ $\epsilon L^{1}(G)^{\wedge}$. But this follows since $\left\{f_{n} g_{n}\right\}_{n=1}^{\infty}$ is a Cauchy sequence in $L^{1}(G)$.

Conversely, for $b \in L^{1}(G)$, write $b=f g, f, g \in L^{2}(G)$. Choose $\left\{\phi_{n}\right\}_{n=1}^{\infty}$, $\left\{\psi_{n}\right\}_{n=1}^{\infty}$ sequences from $\mathcal{C}_{F}(\hat{G})$ such that $\phi_{n} \stackrel{n}{\rightarrow} \hat{f}, \psi_{n} \stackrel{n}{\rightarrow} \hat{g}$ in $\mathfrak{\complement}^{2}(\hat{G})$. Now $\hat{\phi}_{n} \hat{\psi}_{n} \stackrel{n}{\rightarrow} f g$ in $L^{1}(G)$ and so $\hat{b}=(f g)^{\wedge}=\left(\lim _{n \rightarrow \infty} \hat{\phi}_{n} \hat{\psi}_{n}^{n}\right)^{\wedge}=\lim _{n \rightarrow \infty}\left(\hat{\phi}_{n} \hat{\psi}_{n}\right)^{\wedge}=$ $\lim _{n \rightarrow \infty} \phi_{n} \times \psi_{n}=\lim _{n \rightarrow \infty} \phi_{n} \times \lim _{n \rightarrow \infty} \psi_{n} \in \mathscr{\complement}^{2}(\hat{G}) \times \mathfrak{Q}^{2}(\hat{G})$.

2. Multipliers on modules over the Fourier algebra.

Definition. Let $1 / p+1 / q \geq 1, \phi \in \mathfrak{L}^{p}(\hat{G}), \psi \in \mathfrak{L}^{q}(\hat{G})$. We define $\langle\phi, \psi\rangle=$ $(\phi \times \psi)_{\imath}$. This is an extension of $\langle\cdot, \cdot\rangle$ from $\mathcal{C}_{F}(\hat{G}) \times \mathcal{C}_{F}(\hat{G})$.

Definition. Let $1 \leq p, q \leq \infty$. We define $\mathbb{M}_{p, q}=\operatorname{Hom}_{\mathfrak{Q}^{1}(\hat{G})}\left(\mathfrak{\complement}^{p}(\hat{G}), \mathfrak{\complement}^{q}(\hat{G})\right)$, except that we replace $\mathcal{L}^{\infty}(\hat{G})$ by $\mathcal{C}_{0}(\hat{G})$. Note that $\mathfrak{L}^{p}(\hat{G})^{\mathcal{L}^{1}}$ is an $\mathfrak{Q}^{1}(\hat{G})$-module (Corollary 3). (See Rieffel [7] for a more general setting.) 
Proposition 5. Let $T: \mathcal{C}_{F}(\hat{G}) \rightarrow \mathcal{C}_{0}(\hat{G})$ be a linear map. Define $\|T\|_{p, q}=$ $\sup \left\{|\langle T \phi, \psi\rangle|:\|\phi\|_{p} \leq 1,\|\psi\|_{q^{\prime}} \leq 1, \phi, \psi \in \mathcal{C}_{F}(\hat{G})\right\}$. Then $\log \|T\|_{1 / a_{1}, 1 / a_{2}}$ is a convex function for $\left(a_{1}, a_{2}\right) \in[0,1] \times[0,1]$.

Proof. Apply the Riesz-Thorin convexity theorem for integration algebras $[1$, p. 143].

Proposition 6. $\pi_{2,2} \cong L^{\infty}(G)$.

Proof. By taking the inverse Fourier transform we see that $\mathbb{M}_{2,2}$ is isomorphic to the space of bounded maps $T$ from $L^{2}(G)$ to $L^{2}(G)$ which commute with multiplication by elements of $A(G)$, that is, $T: L^{2}(G) \rightarrow L^{2}(G), T(f g)=$ $f(T g), f \in A(G), g \in L^{2}(G)$. Thus $T$ is multiplication by an element of $L^{\infty}(G)$, that is, there exists $b \in L^{\infty}(G)$ such that $T g=b g, g \in L^{2}(G)$ (let $b=T 1$ ).

Theorem 7. Let $1 \leq p, q \leq \infty$. Then $\mathbb{M}_{p, q}=\mathbb{M}_{q^{\prime}, p^{\prime}}$.

Proof. We first suppose $1<p, q<\infty$. Let $T \in \mathbb{M}_{p, q^{\cdot}}$ Thus $T: \mathcal{C}_{F}(\hat{G}) \rightarrow$ $\mathcal{C}_{0}(\hat{G})$, and $\|T\|_{p, q}<\infty$. Now $T(\phi \times \psi)=\phi \times(T \psi), \phi, \psi \in \mathcal{C}_{F}(\hat{G})$. Define the adjoint of $T, S$ by $S: \mathcal{C}_{F}(\hat{G}) \rightarrow \mathcal{C}_{0}(\hat{G})$ and $\langle T \phi, \psi\rangle=\langle\phi, S \psi\rangle, \phi, \psi \in \mathcal{C}_{F}(\hat{G})$. For $\phi, \psi \in \mathcal{C}_{F}(\hat{G}),\langle T \phi, \psi\rangle=((T \phi) \times \psi)_{\imath}=(T(\phi \times \psi))_{\iota}=(T(\psi \times \phi))_{\imath}=((T \psi) \times \phi)_{\imath}$ $=(\phi \times(T \psi))_{l}=\langle\phi, T \psi\rangle$. Thus $S$ and $T$ agree on $\mathcal{C}_{F}(\hat{G})$.

Now for $\phi, \psi \in \mathcal{C}_{F}(\hat{G}),\langle T \phi, \psi\rangle=\langle\phi, S \psi\rangle=\langle\phi, T \psi\rangle$, and so $\|T\|_{p, q}=$ $\|T\|_{q^{\prime}, p^{\prime}}$. It follows that $T \mid \mathcal{C}_{F}(\hat{G})$ extends uniquely to an element of $\pi_{q^{\prime}, p^{\prime}}^{\prime}$ and so $\pi_{p, q} \subset \Re_{q^{\prime}, p^{\prime}}$. By symmetry $\Re_{q^{\prime}, p^{\prime}}=\pi_{p, q^{\prime}}$.

We consider now the exceptional cases. Since $\mathcal{L}^{1}(\hat{G})$ has an identity, we obtain $\mathbb{M}_{1, p}=\mathscr{L}^{p}(\hat{G})$ for $1 \leq p<\infty$ and $\mathbb{M}_{1, \infty}=\mathcal{C}_{0}(\hat{G})$. Further, applying the previous argument we see that $T \in \mathbb{M}_{p, \infty}$ implies $T \in \mathbb{M}_{1, p^{\prime}}=\mathscr{L}^{\prime}(\hat{G})$. But by Corollary $3, \mathfrak{Q}^{p^{\prime}}(\hat{G}) \subset \mathbb{M}_{p, \infty}$, so $\mathbb{M}_{p, \infty}=\mathbb{M}_{1, p^{\prime}}$. The other spaces $\mathbb{M}_{p, 1}(p>1)$ and $M_{\infty, q}(q<\infty)$ will be shown to be trivial in Theorem 10 .

Theorem 8. Let $1<p<q<2$. Then

$$
A(G) \cong \mathscr{L}^{1}(\hat{G})=M_{1,1} \subset \mathbb{M}_{p, p} \subset \mathbb{M}_{q, q} \subset \mathbb{M}_{2,2} \cong L^{\infty}(G) .
$$

Proof. That $\mathscr{L}^{1}(\hat{G})=\mathbb{M}_{1,1}$ follows since $A(G)$ has an identity.

Since $\mathscr{L}^{p}(\hat{G})$ is an $\mathscr{L}^{1}(\hat{G})$-module, $\mathbb{M}_{1,1} \subset \mathbb{M}_{p, p}$ (recall Theorem 2).

Let $T \in \mathbb{M}_{q, q^{*}}$. Then $\|T\|_{q, q}=\|T\|_{q^{\prime}, q^{\prime}<\infty}$. Since $\log \|T\|_{1 / a_{1,1 / a_{2}}}$ is a convex function of $\left(a_{1}, a_{2}\right) \in[0,1] \times[0,1],\|T\|_{2,2} \leq\|T\|_{q, q^{*}}$ 'Thus $\dot{\pi}_{q, q} \subset \mathbb{M}_{2,2}$.

Now for $T \in \mathbb{M}_{p, p},\|T\|_{p, p}<\infty$. A lso $\|T\|_{2,2} \leq\|T\|_{p, p}<\infty$. Now since $1 / 2<1 / q<1 / p$, we can interpolate to get $\|T\|_{q, q} \leq\|T\|_{p, p}<\infty$. Thus $\pi_{p, p} \subset \pi_{q, q}$.

Theorem 9. Let $1 \leq p<2$. Then $\mathbb{M}_{p, p} \neq \mathbb{M}_{2,2}$. 
Proof. By way of contradiction, suppose $\mathbb{M}_{p, p}=\mathbb{M}_{2,2}=L^{\infty}(G)^{\wedge}$. Then $L^{\infty}(G)^{\wedge} \subset \mathscr{L}^{p}(\hat{G})$ (since $\left.\hat{1} \in \mathfrak{L}^{p}(\hat{G})\right)$, and so $\|\hat{f}\|_{p} \leq C\|f\|_{\infty}, f \in L^{\infty}(G), C<\infty$. In particular, $f \mapsto \hat{f}$ maps $C(G)$ into $\mathscr{Q}^{p}(\hat{G})$, and its adjoint $\Upsilon$ maps $\mathfrak{Q}^{p}(\hat{G})$ into $M(G)$. Further $\Upsilon: \mathscr{Q}^{p^{\prime}}(\hat{G}) \rightarrow L^{1}(G)$ (since $\Upsilon\left(\mathcal{C}_{F}(\hat{G})\right) \subset L^{1}(G)$ and $L^{1}(G)$ is closed). Let $\phi \in \mathscr{L}^{p^{\prime}}(\hat{G})$ and $\omega \in \mathscr{L}^{\infty}(\hat{G})$. Then $\phi \omega \in \mathscr{L}^{p^{\prime}}(\hat{G})$ and $(\phi \omega)^{\wedge} \epsilon$ $L^{1}(G)$, that is, the map $\omega \mapsto(\phi \omega)^{\wedge}$ takes $\mathcal{L}^{\infty}(\hat{G})$ into $L^{1}(G)$. It follows now from a theorem of S. Helgason $\left[5\right.$, p. 785] that $\phi \in \mathscr{L}^{2}(\hat{G})$. Thus $\mathscr{L}^{\prime}(\hat{G}) \subset \mathscr{L}^{2}(\hat{G})$, a contradiction.

Theorem 10. Let $1 \leq p<q \leq \infty$, then $\mathbb{M}_{q, p}=\{0\}$.

Proof. First, let $1<p^{\prime}<2<p$. We show that $\mathbb{M}_{p, p^{\prime}}=\{0\}$. For if $T \in \mathbb{M}_{p, p^{\prime}}, T \neq 0$, then there exists $b \in L^{\infty}(G), b \neq 0$, such that $f \mapsto b f$ is a bounded linear operator from $L^{p^{\prime}}(G) \rightarrow L^{p}(G)$ (consider the maps: $L^{p^{\prime}}(G) \stackrel{\hat{\rightarrow}}{ }$ $\mathfrak{Q}^{p}(\hat{G}) \stackrel{T}{\rightarrow} \mathscr{L}^{p}(\hat{G}) \hat{\rightarrow} L^{p}(G)$, see $[1$, p. 144]). Thus there exists $C<\infty$ such that $\|b f\|_{p} \leq C\|f\|_{p^{\prime}}, f \in L^{p^{\prime}}(G)$. Let $\epsilon>0$ be such that $\{x:|b(x)| \geq \epsilon\}$ contains a measurable set $E$ with $m_{G}(E)>0$, and let $\chi_{E}$ denote the characteristic function of $E$. Then

$$
\epsilon^{p} m_{G}(E) \leq\left\|b \chi_{E}\right\|_{p}^{p} \leq C^{p}\left\|\chi_{E}\right\|_{p^{\prime}}^{p}=C^{p}\left(m_{G}(E)\right)^{p / p^{\prime}},
$$

and so $0<\epsilon^{p} / C^{p} \leq\left(m_{G}(E)\right)^{p / p^{-1}-1}$. But let $m_{G}(E)$ tend to 0 for the required contradiction. Thus we have established $M_{p, p^{\prime}}=\{0\}, 1<p^{\prime}<2<p$.

Now let $T \in \mathbb{M}_{q, p}, T \neq 0,1 \leq p<q \leq \infty$, excepting the case $\mathbb{M}_{\infty, 1}$. Thus, $\|T\|_{p^{\prime}, q^{\prime}}=\|T\|_{q, p}<\infty$. The Riesz-Thorin convexity theorem implies for $1 / r=$ $1 / 2-1 / 2 p+1 / 2 q$ that $\mathbb{M}_{r, r^{\prime}} \neq\{0\}$, a contradiction. Finally, $\mathbb{M}_{\infty, 1} \subset \mathbb{M}_{2,1}=\{0\}$.

Remark. The proof of the above theorem was suggested to us by our colleague John Fournier.

3. Multipliers as dual spaces. For $G$ abelian, $\Re_{p, p}$ is the space of $L^{p}(\hat{G})$ multipliers, and A. Figà-Talamanca [4] (also M. Rieffel [7]) has shown it to be a dual space. We now will exhibit this result for the case of $G$ nonabelian (compact). For $p=1, \pi_{1,1}$ is clearly a dual space; indeed, $\pi_{1,1}=\mathscr{L}^{1}(\hat{G})=$ $\mathcal{C}_{0}(\hat{G})^{*}$ (see $[1$, p. 88]).

Definition. Let $1<p \leq 2$. For $\phi \in \mathcal{C}_{0}(\hat{G})$, we define

$$
\begin{aligned}
& \|\phi\|_{p}=\inf \left\{\sum_{n=1}^{\infty}\left\|\phi_{n}\right\|_{p}\left\|\psi_{n}\right\|_{p^{\prime}}: \phi=\sum_{n=1}^{\infty} \phi_{n} \times \psi_{n} \text { (convergence in } \mathcal{C}_{0}(\hat{G})\right), \\
& \left.\left\{\phi_{n}\right\}_{n=1}^{\infty} \subset \mathfrak{L}^{p}(\hat{G}),\left\{\psi_{n}\right\}_{n=1}^{\infty} \subset \mathcal{L}^{p^{\prime}}(\hat{G})\right\} \text {. }
\end{aligned}
$$

We use the convention that inf $\varnothing=\infty$. The subspace of $\mathcal{C}_{0}(\hat{G})$ consisting of all $\phi$ with $\|\phi\|_{p}<\infty$ is denoted by $\mathbb{Q}_{p}$. 
Remark. By Theorem 4, $\mathbb{Q}_{2}=L^{1}(G)^{\wedge}$.

Proposition 11. For $1<p \leq 2, \mathbb{A}_{p}$ is a Banach space.

Proof. It is easy to show $\|\cdot\|_{p}$ is a norm. We wish now to show that $\mathbb{C}_{p}$ is complete with respect to $\|\cdot\|_{p}$. Let $\left\{\phi_{n}\right\}_{n=1}^{\infty}$ be a Cauchy sequence in $\mathbb{A}_{p}$. We may assume that $\left\|\phi_{n}-\phi_{n+1}\right\|_{p}<1 / 2^{n+1}$. Let $\psi_{n}=\phi_{n+1}-\phi_{n} \in \mathbb{Q}_{p}$, and so write $\psi_{n}$ as $\sum_{m=1}^{\infty} \theta_{n m} \times \omega_{n m}, \theta_{n m} \in \mathscr{L}^{p}(\hat{G}), \omega_{n m} \in \mathscr{L}^{p^{\prime}}(\hat{G})$, and $\Sigma_{m=1}^{\infty}\left\|\theta_{n m}\right\|_{p}\left\|\omega_{n m}\right\|_{p^{\prime}}<1 / 2^{n}$. Let $\phi=\phi_{1}+\sum_{n=1}^{\infty} \psi_{n}$. Now $\|\phi\|_{p} \leq\left\|\phi_{1}\right\|_{p}+$ $\sum_{n=1}^{\infty} 1 / 2^{n}<\infty$, and so $\phi \in \mathbb{P}_{p}$. Also $\left\|\phi_{m}-\phi\right\|_{p}=\left\|\sum_{n=m+1}^{\infty} \psi_{n}\right\|_{p}<\sum_{n=m+1}^{\infty} 1 / 2^{n}$, which is small for large enough $m$.

Theorem 12. Let $\xi \in \mathbb{P}_{p}^{*}(1<p \leq 2)$. Then there exists $T \in \mathbb{M}_{p, p}$ such that $\|T\|_{p, p} \leq\|\xi\|$ and $\langle T \phi, \psi\rangle=\xi(\phi \times \psi), \phi, \psi \in \mathcal{C}_{F}(\hat{G})$.

Proof. For $\phi, \psi \in \mathcal{C}_{F}(\hat{G}),|\xi(\phi \times \psi)| \leq\|\phi \times \psi\|_{p}\|\xi\| \leq\|\phi\|_{p}\|\psi\|_{p} \cdot\|\xi\|$. Thus, for each $\phi \in \mathcal{C}_{F}(\hat{G})$, the map $\psi \mapsto \xi(\phi \times \psi)$ extends to a bounded linear functional on $\mathfrak{L}^{p^{\prime}}(\hat{G})$. Let $\omega \in \mathfrak{L}^{p}(\hat{G})=\left(\mathfrak{Q}^{\prime}(\hat{G})\right)^{*}$ be such that $\langle\omega, \psi\rangle=\xi(\phi \times \psi)$. Define $T \phi=\omega\left(\phi \in \mathcal{C}_{F}(\hat{G})\right)$. Thus $\langle T \phi, \psi\rangle=\xi(\phi \times \psi)$. Now $T: \mathcal{C}_{F}(\hat{G}) \rightarrow \mathfrak{Q}^{p}(\hat{G})$ and $\|T\|_{p, p} \leq\|\xi\|$, so we may extend $T$ to all of $\mathcal{L}^{p}(\hat{G})$. Finally, to see that $T \in \mathbb{M}_{p, p}$ we note that $\left\langle T\left(\phi_{1} \times \phi_{2}\right), \psi\right\rangle=\xi\left(\left(\phi_{1} \times \phi_{2}\right) \times \psi\right)=\xi\left(\phi_{1} \times\left(\phi_{2} \times \psi\right)\right)=$ $\left(\left(T \phi_{1}\right) \times\left(\phi_{2} \times \psi\right)\right)_{\imath}=\left\langle\left(T \phi_{1}\right) \times \phi_{2}, \psi\right\rangle, \phi_{1}, \phi_{2}, \psi \in \mathcal{C}_{F}(\hat{G})$. Thus $T\left(\phi_{1} \times \phi_{2}\right)=$ $\left(T \phi_{1}\right) \times \phi_{2}, \phi_{1}, \phi_{2} \in \mathcal{C}_{F}(\hat{G})$. Thus $T \in \mathbb{M}_{p, p}$.

Proposition 13. Let $\phi \in \mathfrak{L}^{p}(\hat{G})(1 \leq p<\infty)$ or $\phi \in \mathcal{C}_{0}(\hat{G})(p=\infty)$ and $\epsilon>0$. Then there exists a sequence $\left\{\phi_{n}\right\}_{n=1}^{\infty} \subset \mathcal{C}_{F}(\hat{G})$ such that $\sum_{n=1}^{\infty}\left\|\phi_{n}\right\|_{p}<\|\phi\|_{p}+\epsilon$ and $\sum_{n=1}^{\infty} \phi_{n}=\phi$ (convergence in norm).

Proof. For $n=1,2, \cdots$, let $\psi_{n} \in \mathcal{C}_{F}(\hat{G})$ be such that $\left\|\psi_{n}-\phi\right\|_{p}<\epsilon / 2^{n}$. Let $\phi_{1}=\psi_{1}$ and, for $n=2,3, \ldots$, let $\phi_{n}=\psi_{n+1}-\psi_{n}$. Then $\left\{\phi_{n}\right\}_{n=1}^{\infty} \subset$ $\mathcal{C}_{F}(\hat{G}), \sum_{n=1}^{\infty}\left\|\phi_{n}\right\|_{p}<\|\phi\|_{p}+\sum_{n=1}^{\infty} \epsilon / 2^{n}=\|\phi\|_{p}+\epsilon$, and $\sum_{n=1}^{N} \phi_{n}=\psi_{N+1} \stackrel{N}{\rightarrow} \phi$ in $\mathcal{L}^{p}(\hat{G})$.

Proposition 14. Let $\phi \in \mathfrak{L}^{p}(\hat{G}), \psi \in \mathfrak{L}^{p^{\prime}}(\hat{G})$, and $\epsilon>0(1<p \leq 2)$. Then there $2 x$ ist sequences $\left\{\phi_{n}\right\}_{n=1}^{\infty},\left\{\psi_{n}\right\}_{n=1}^{\infty} \subset \mathcal{C}_{F}(\hat{G})$ such that $\sum_{n=1}^{\infty}\left\|\phi_{n}\right\|_{p}\left\|\psi_{n}\right\|_{p} \cdot<$ $\|\phi\|_{p}\|\psi\|_{p^{\prime}}+\epsilon$, and $\sum_{n=1}^{\infty} \phi_{n} \times \psi_{n}=\phi \times \psi$ (convergence in $\mathcal{C}_{0}(\hat{G})$ ).

Proof. Let $\epsilon^{\prime}, \epsilon^{\prime \prime}>0$ be chosen in a way to be specified later. By Proposition 13, there exist sequences $\left\{\phi_{n}\right\}_{n=1}^{\infty},\left\{\psi_{n}\right\}_{n=1}^{\infty} \subset \mathcal{C}_{F}(\hat{G})$ such that $\Sigma_{n=1}^{\infty} \phi_{n}=\phi, \Sigma_{n=1}^{\infty} \psi_{n}=\psi, \Sigma_{n=1}^{\infty}\left\|\phi_{n}\right\|_{p}<\|\phi\|_{p}+\epsilon^{\prime}$, and $\sum_{n=1}^{\infty}\left\|\psi_{n}\right\|_{p^{\prime}}<\|\psi\|_{p^{\prime}+}$ $\epsilon^{\prime \prime}$. Let $\phi_{n}^{\prime}=\sum_{k=1}^{n} \phi_{k}$ and $\psi_{n}^{\prime}=\Sigma_{k=1}^{n} \psi_{k}$. Now $\phi_{n}^{\prime} \times \psi_{n}^{\prime} \stackrel{n}{\rightarrow} \phi \times \psi$ in $\mathcal{C}_{0}(\hat{G})$ (by joint continuity). Now $\phi_{n}^{\prime} \times \psi_{n}^{\prime}=\sum_{k, l=1}^{n} \phi_{k} \times \psi_{l}$; also $\sum_{k, l=1}^{n}\left\|\phi_{k}\right\|_{p}\left\|\psi_{l}\right\|_{p^{\prime}}$ $=\Sigma_{k=1}^{n}\left\|\phi_{k}\right\|_{p} \Sigma_{l=1}^{n}\left\|\psi_{l}\right\|_{p^{\prime}}<\left(\|\phi\|_{p}+\epsilon^{\prime}\right)\left(\|\psi\|_{p^{\prime}}+\epsilon^{\prime \prime}\right)<\|\phi\|_{p}\|\psi\|_{p^{\prime}}+\epsilon$ for the 
appropriate choice of $\epsilon^{\prime}, \epsilon^{\prime \prime}$. Finally, note that $\phi_{n}^{\prime} \times \psi_{n}^{\prime}=\sum_{k, l=1}^{n} \phi_{k} \times \psi_{l}$.

Proposition 15. Let $\omega \in \mathbb{Q}_{p}(1<p \leq 2)$ and $\epsilon>0$. Then there exist sequences $\left\{\phi_{n}\right\}_{n=1}^{\infty},\left\{\psi_{n}\right\}_{n=1}^{\infty} \subset \mathcal{C}_{F}(\hat{G})$ such that $\omega=\sum_{n=1}^{\infty} \phi_{n} \times \psi_{n}$ (convergence in $\left.\mathcal{C}_{0}(\hat{G})\right)$ and $\sum_{n=1}^{\infty}\left\|\phi_{n}\right\|_{p}\left\|\psi_{n}\right\|_{p^{\prime}}<\|\omega\|_{p}+\epsilon$.

Proof. There exist sequences $\left\{\phi_{n}^{\prime}\right\}_{n=1}^{\infty} \subset \mathscr{L}^{p}(\hat{G})$ and $\left\{\psi_{n}^{\prime}\right\}_{n=1}^{\infty} \subset \mathscr{L}^{\prime}(\hat{G})$ such that $\omega=\sum_{n=1}^{\infty} \phi_{n}^{\prime} \times \psi_{n}^{\prime}$ and $\sum_{n=1}^{\infty}\left\|\phi_{n}^{\prime}\right\|_{p}\left\|\psi_{n}^{\prime}\right\|_{p^{\prime}}<\|\omega\|_{p}+\epsilon^{\prime} 2$. For each $n=1,2$, $\cdots$, there exist sequences $\left\{\phi_{n m}\right\}_{m=1}^{\infty},\left\{\psi_{n m}\right\}_{m=1}^{\infty} \subset C_{F}(\hat{G})$ such that $\phi_{n} \times \psi_{n}=$ $\sum_{m=1}^{\infty} \phi_{n m} \times \psi_{n m}$ and $\sum_{m=1}^{\infty}\left\|\phi_{n m}\right\|_{p}\left\|\psi_{n m}\right\|_{p^{\prime}}<\left\|\phi_{n}^{\prime}\right\|_{p}\left\|\psi_{n}^{\prime}\right\|_{p^{\prime}}+\epsilon / 2^{n+1}$. Now $\sum_{n=1}^{\infty} \sum_{m=1}^{\infty}\left\|\phi_{n m}\right\|_{p}\left\|\psi_{n m}\right\|_{p^{\prime}}<\sum_{n=1}^{\infty}\left\|\phi_{n}^{\prime}\right\|_{p}\left\|\psi_{n}^{\prime}\right\|_{p}^{\prime}+\epsilon / 2<\|\omega\|_{p}+\epsilon$ and $\sum_{n=1}^{\infty} \sum_{m=1}^{\infty} \phi_{n m} \times \psi_{n m}=\omega$.

Proposition 16. Let $\delta>0$ and let $X_{\delta}=\left\{\omega \in \mathcal{C}_{F}(\hat{G}): \omega=\Sigma_{n=1}^{N} \phi_{n} \times \psi_{n}\right.$, $\phi_{n}, \psi_{n} \in \mathcal{C}_{F}(\hat{G}),\|\omega\|_{p}+\delta>\sum_{n=1}^{N}\left\|\phi_{n}\right\|_{p}\left\|\psi_{n}\right\|_{p}$, some $\left.N=1,2, \ldots\right\}$. Then each $X_{\delta}$ is dense in $\mathbb{Q}_{p}(1<p \leq 2)$.

Proof. Fix $\delta>0, \xi \in \mathbb{A}_{p}$, and $0<\epsilon<\delta / 2$. By Proposition 15, there exist sequences $\left\{\phi_{n}\right\}_{n=1}^{\infty},\left\{\psi_{n}\right\}_{n=1}^{\infty} \subset \mathcal{C}_{F}(\hat{G})$ such that $\xi=\sum_{n=1}^{\infty} \phi_{n} \times \psi_{n}$ and $\sum_{n=1}^{\infty}\left\|\phi_{n}\right\|_{p}\left\|\psi_{n}\right\|_{p^{\prime}}<\|\xi\|_{p}+\epsilon$. Choose $N$ such that $\sum_{n=N+1}^{\infty}\left\|\phi_{n}\right\|_{p}\left\|\psi_{n}\right\|_{p^{\prime}}<\epsilon$ and let $\omega=\sum_{n=1}^{N} \phi_{n} \times \psi_{n}$. Then $\|\omega-\xi\| \leq\left\|\Sigma_{n=N+1}^{\infty} \phi_{n} \times \psi_{n}\right\|<\epsilon$ and $\sum_{n=1}^{N}\left\|\phi_{n}\right\|_{p}\left\|\psi_{n}\right\|_{p^{\prime}} \leq \sum_{n=1}^{\infty}\left\|\phi_{n}\right\|_{p}\left\|\psi_{n}\right\|_{p^{\prime}}<\|\xi\|_{p}+\epsilon \leq\|\omega\|_{p}+2 \epsilon$. Thus $\omega \epsilon X_{\delta}$ and $\|\omega-\xi\|_{p}<\epsilon$.

Theorem 17. Let $1<p \leq 2$ and $T \in \mathbb{M}_{p, p^{*}}$. Then $T$ extends to a bounded linear map from $\mathfrak{A}_{p} \rightarrow \mathbb{Q}_{p}$, and the linear functional $T^{\#}: \mathbb{A}_{p} \rightarrow C$ given by $T^{\#}(\omega)=(T \omega)_{l}$ is in $\mathbb{A}_{p}^{*}$ with $\left\|T^{\#}\right\| \leq\|T\|$. Thus $\mathbb{A}_{p}^{*} \cong \mathbb{M}_{p, p}$.

Proof. Let $\delta>0$ and $\omega \in X_{\delta} \subset \mathcal{C}_{F}(\hat{G}) \subset \mathfrak{Q}^{p}(\hat{G})$. Write $\omega=\sum_{n=1}^{N} \phi_{n} \times \psi_{n}$, $\phi_{n}, \psi_{n} \in \mathcal{C}_{F}(\hat{G})$, where $\|\omega\|_{p}+\delta>\Sigma_{n=1}^{N}\left\|\phi_{n}\right\|_{p}\left\|\psi_{n}\right\|_{p^{\prime}}$. Now $T \omega=T\left(\sum_{n=1}^{N} \phi_{n} \times \psi_{n}\right)$ $=\sum_{n=1}^{N} T\left(\phi_{n} \times \psi_{n}\right)=\Sigma_{n=1}^{N}\left(T \phi_{n}\right) \times \psi_{n}$, and $\|T \omega\|_{p} \leq \sum_{n=1}^{N}\left\|T \phi_{n}\right\|_{p}\left\|\psi_{n}\right\|_{p}^{\prime} \leq$ $\|T\|_{p, p}\left(\|\omega\|_{p}+\delta\right)$. But $X_{\delta}$ is dense in $\mathbb{Q}_{p}$ and so $T$ extends to $\mathbb{C}_{p}$ with norm less than or equal to $\|T\|_{p, p}(1+\delta)$. But $\delta>0$ is arbitrary and so $\|T \omega\|_{p} \leq\|T\|_{p, p}\|\omega\|_{p}$.

Corollary 18. For $1 \leq r<2, \mathbb{M}_{r, r} \subsetneq \bigcap\left\{\mathbb{M}_{s, s}: r<s<2\right\}$, and for $1<r \leq 2$, $\bigcup\left\{\mathbb{M}_{s, s}: 1<s<r\right\} \varsubsetneqq \mathbb{M}_{r, r}$

Proof. J. F. Price [6, pp. 326-330] has given a general argument based on the Riesz-Thorin convexity theorem which yields the corollary using only the facts that $\pi_{q, q} \neq \pi_{2,2}(q<2)$ (see Theorem 9$)$, that $\pi_{q, q}$ is the dual space of $\mathfrak{Q}_{q}$, and that $\stackrel{\mathbb{Q}}{q}_{q}$ contains $\mathscr{L}^{1}(\hat{G})$ as a dense subspace (see Proposition 16).

Definition. Let $1 \leq p, q<\infty, 1 / p+1 / q \geq 1$, and $1 / r=1 / p+1 / q-1$. We define for $\phi \in \mathfrak{Q}^{r}(\hat{G})$, 


$$
\begin{aligned}
\|\phi\|_{p, q}=\inf \left\{\sum_{n=1}^{\infty}\left\|\phi_{n}\right\|_{p}\left\|\psi_{n}\right\|_{q}: \phi=\right. & \left.\sum_{n=1}^{\infty} \phi_{n} \times \psi_{n} \text { (convergence in } \mathcal{L}^{r}(\hat{G})\right), \\
& \left.\left\{\phi_{n}\right\}_{n=1}^{\infty} \subset \mathfrak{L}^{p}(\hat{G}),\left\{\psi_{n}\right\}_{n=1}^{\infty} \subset \mathscr{L}^{q}(\hat{G})\right\} .
\end{aligned}
$$

The subspace of $\mathcal{L}^{r}(\hat{G})$ consisting of all $\phi$ with $\|\phi \mid\|_{p, q}<\infty$ is denoted by $\mathbb{Q}_{p, q}$.

Remark. For $1<p<\infty$, observe that $\mathfrak{Q}_{p, p^{\prime}}=\mathbb{Q}_{p}$; and indeed, for $1 \leq p<$ $q \leq \infty$, one can show that $\mathbb{Q}_{p, q^{\prime}}^{*} \cong \mathbb{M}_{p, q}$, by appropriately modifying the preceding proofs. (Note for $p>q$ that $\Re_{p, q}=\{0\}$, and for $1 \leq p<q \leq \infty$ that $1 / p+1 / q^{\prime}>1$.)

Definition. Let $W O$ denote the weak operator topology on $\Re_{p, p}$, and let $w^{*}$ denote the weak- ${ }^{*}$ topology on $\mathbb{M}_{p, p}(1<p \leq 2)$ from the pairing of $\mathbb{C}_{p}$ with $M_{p, p}$. Thus $T_{a} \stackrel{a}{\rightarrow} T\left(\{T\},\{T\} \subset M_{p, p}^{p}\right)$ in $W O$ if and only if $\left\langle T_{a} \phi, \psi\right\rangle \stackrel{a_{a}}{\rightarrow}\langle T \phi, \psi\rangle$,

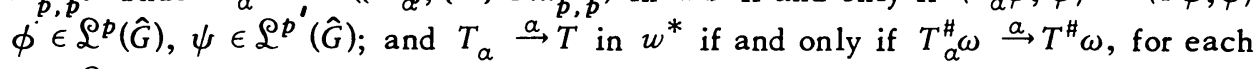
$\omega \in \mathbb{P}_{p}$.

Theorem 19. In $\pi_{p, p}(1<p \leq 2)$, WO $\subset w^{*}$.

Proof. Let $T_{a}, T \in \mathbb{M}_{p, p}$ with $T_{a} \stackrel{a}{\rightarrow} T$ in $w^{*}$. Thus $T_{a}^{\#} \omega \stackrel{a}{\rightarrow} T^{\#} \omega$ for all $\omega \in \mathbb{Q}_{p}$. Extend $T_{a}, T$ to operators from $\mathbb{Q}_{p}$ to $\mathbb{Q}_{p}$ (as in Theorem 17 ) such that $T_{a}^{\#} \omega=\left(T_{\alpha} \omega\right)_{\imath}, T^{\#} \omega=(T \omega)_{\iota}\left(\omega \in \mathbb{Q}_{p}\right)$. Let $\phi \in \mathscr{L}^{p}(\hat{G}), \psi \in \mathfrak{L}^{p}{ }^{\prime}(\hat{G})$. We wish to show that $\left\langle T_{a} \phi, \psi\right\rangle \stackrel{a}{\rightarrow}\langle T \phi, \psi\rangle$. It suffices to show that $S(\phi \times \psi)=$ $(S \phi) \times \psi, S \in \mathbb{M}_{p, p}:$ for then $\left\langle T_{a} \phi, \psi\right\rangle=\left(\left(T_{a} \phi\right) \times \psi\right)_{l}=\left(T_{a}(\phi \times \psi)\right)_{l}=$ $T_{a}^{\#}(\phi \times \psi) \stackrel{a}{\rightarrow} T^{\#}(\phi \times \psi)=(T(\phi \times \psi))_{\iota}=((T \phi) \times \psi)_{\iota}=\langle T \phi, \psi\rangle$. Now let $\psi_{n} \stackrel{n}{\rightarrow} \psi$ in $\mathcal{L}^{\prime}(\hat{G}),\left\{\psi_{n}\right\}_{n=1}^{\infty} \subset \mathcal{C}_{F}(\hat{G})$. Then for $S \in \mathbb{M}_{p, p}$, we have that $\phi \times \psi_{n} \stackrel{n}{\rightarrow} \phi \times \psi$ in $\mathbb{Q}_{p}$ and so $S(\phi \times \psi)=\lim _{n \rightarrow \infty} S\left(\phi \times \psi_{n}\right)=\lim _{n \rightarrow \infty}(S \phi) \times \psi_{n}$ $=(S \phi) \times \psi$.

Corollary 20. On bounded subsets of $\mathbb{M}_{p, p}(1<p \leq 2), w^{*}=$ WO.

Proof. Bounded closed subsets of $\mathbb{Q}_{p}^{*} \cong \mathbb{M}_{p, p}$ are $w^{*}$-compact.

Theorem 21. Let $\Phi$ denote the $w^{*}$-closure of $\mathcal{C}_{F}(\hat{G})$ or $\mathscr{L}^{1}(\hat{G})$ in $\mathbb{M}_{p, p}$, $1<p<\infty$. Then $\Phi=\mathbb{M}_{p, p}$.

Proof. Suppose $\Phi \neq \mathbb{M}_{p, p}$, then there exists $\omega \in \mathbb{Q}_{p}$ such that $\omega \neq 0$ and $T^{\#}(\omega)=0$ for all $T \in \mathcal{C}_{F}(\hat{G}) \subset \mathbb{M}_{p, p}$. But if $T \in \mathcal{C}_{F}(\hat{G})$, considered as a subspace of $\mathbb{M}_{p, p}$, then there exists a $\phi \in \mathcal{C}_{F}(\hat{G})$ such that $T \psi=\phi \times \psi$ for all $\psi \in \mathfrak{Q}^{p}(\hat{G})$. Thus $T^{\#}(\omega)=(T \omega)_{\iota}=(\phi \times \omega)_{i}=\langle\phi, \omega\rangle=0$, for all $\phi \in \mathcal{C}_{F}(\hat{G})$. But $\omega \in \mathbb{Q}_{p} \subset \mathcal{C}_{0}(\hat{G})$, so $\omega=0$.

Corollary 22. For $1<p<\infty, \mathcal{C}_{F}(\hat{G})$ is WO-dense in $\pi_{p, p^{*}}$

Remark. An invariant mean on $\mathscr{L}^{\infty}(\hat{G})$ is a bounded linear functional $p$ on 
$\mathcal{L}^{\infty}(\hat{G})$ such that $(1) p(\phi) \geq 0$ whenever $\phi \geq 0,(2) p(I)=1$ ( $I$ is the identity in $\left.\mathfrak{L}^{\infty}(\hat{G})\right)$, and (3) $p(\hat{f} \times \phi)=f(e) p(\phi), f \in A(G), \phi \in \mathfrak{L}^{\infty}(\hat{G})$. In [2] we showed that invariant means exist on $\mathfrak{L}^{\infty}(\hat{G})$.

Let $p$ be an invariant mean on $\mathscr{L}^{\infty}(\hat{G})$. Define $T: \mathscr{L}^{\infty}(\hat{G}) \rightarrow \mathscr{L}^{\infty}(\hat{G})$ by $\langle\psi, T \phi\rangle=p(\psi \times \phi), \psi \in \mathfrak{L}^{1}(\hat{G}), \phi \in \mathfrak{L}^{\infty}(\hat{G}) ;$ and so $T \phi=p(\phi) I$. Thus $T \epsilon$

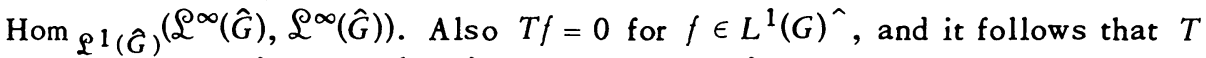
annihilates $\mathcal{C}_{0}(\hat{G})=\mathrm{cl}\left(L^{1}(G)^{\wedge}\right)$ (closure in $\mathcal{C}_{0}(\hat{G})$ ): since for $\mu \in M(G)$, $p(\hat{\mu})=\mu(\{e\})($ see $[3])$.

\section{BIBLIOGRAPHY}

1. C. Dunkl and D. Ramirez, Topics in harmonic analysis, Appleton-Century-Crofts, New York, 1971.

2. - Existence and nonuniqueness of invariant means on $\mathfrak{L}^{\infty}(\hat{G})$, Proc. Amer. Math. Soc. 32 (1972), 525-530.

3. - Helson sets in compact and locally compact groups, Michigan Math. J. 19 (1972), 65-69.

4. A. Figà-Talamanca, Translation invariant operators in $L^{p}$, Duke Math. J. 32 (1965), 495-501. MR $31 \# 6095$.

5. S. Helgason, Lacunary Fourier series on noncommutative groups, Proc. Amer. Math. Soc. 9 (1958), 782-790. MR 20 \#6667.

6. J. Price, Some strict inclusions between spaces of $L^{p}$-multipliers, Trans. Amer. Math. Soc. 152 (1970), 321-330.

7. M. Rieffel, Multipliers and tensor products of $L^{p}$-spaces of locally compact groups, Studia Math. 33 (1969), 71-82. MR 39 \#6078.

DEPARTMENT OF MATHEMATICS, UNIVERSITY OF VIRGINIA, CHARLOTTESVILLE, VIRGINIA 22903 